\title{
Thermodynamic Volume in AdS/CFT
}

\author{
Kyung Kiu Kim ${ }^{1, \star}$ and Byoungjoon Ahn ${ }^{2, \star \star}$ \\ ${ }^{1}$ Department of Physics and Astronomy, Sejong University, Seoul 05006, Korea \\ ${ }^{2}$ Department of Physics, College of Science, Yonsei University, Seoul 03722, Korea
}

\begin{abstract}
In this note, we study on extended thermodynamics of AdS black holes by varying cosmological constant. We found and discussed pressure and volume of both bulk and boundary physics through AdS/CFT correspondence. In particular, we derive the relation between thermodynamic volume and a chemical potential for M2 brane dual to four dimensional AdS space. In addition, we show that thermodynamic volume of hyperbolic black hole is related to 'entanglement pressure' coming from a generalized first law of entanglement entropy.
\end{abstract}

\section{Introduction and Summary}

Thermodynamics of black hole is the starting point of all controversies in quantum gravity. It has given rise to many puzzles, new approaches and fusion researches among various areas in physics. One of the approaches supported by most people is the holographic principle [1,2]. Maldacena found that this principle can be realized exactly with various backgrounds in string theory[3]. This correspondence was extended to the black holes immediately[4]. Thus the thermodynamics of black holes is identified with thermodynamics of conformal field theories through AdS/CFT correspondence.

Recently there have been meaningful attempts to extend thermodynamics of black holes. It is simply to consider a pressure of the bulk thermodynamics [5]. To this end, the cosmological constant can be regarded as a thermodynamic variable, pressure. It is quite natural, since the cosmological constant plays a role of energy-momentum in the bulk. Under this generalization, the mass of black hole is interpreted as enthalpy rather than internal energy. Moreover, a van der Waals like critical point appears and provides a richer phase structure. [6-8]

Smarr relation shows the effect of the pressure directly even for the fixed cosmological constant, while The first law of black hole thermodynamics doesn't give us any sign of the pressure without varying cosmological constant. Furthermore, it reveals the conjugate (thermodynamic) volume to the pressure and gives new interpretation of the black hole mass as an enthalpy. In AdS space, there are three kinds of non-rotating black holes, such as black brane, black hole and black hole with hyperbolic spatial section. They share a universal form of Smarr relation [9].

On the other hand, these three sorts of black holes are dual to finite temperature conformal field theories in flat space, spherical space and hyperbolic space, respectively, through AdS/CFT correspondence. Nonvanishing curvature of space corrects the energy-momentum tensor of the dual field theory

\footnotetext{
$\star$ e-mail: kimkyungkiu@ sejong.ac.kr

$\star \star$ e-mail: bjahn@yonsei.ac.kr
} 
with Casimir contribution. Smarr relation in the bulk can be written down in terms of on-shell action and boundary quantities shch as energy density, entropy, temperature, charge density and chemical potential. From this relation one can notice that the gravity on-shell action is nothing but the grand potential of the dual boundary system. By varying this relation and adopting on-shell condition, the first law of the dual theory can be derived. Also, the pressure of the dual system can be read off from this. we will provide summary of the results in the next section.

Physical interpretation of the thermodynamic volume depends on how the cosmological constant changes physically. For the black holes with spherical and flat spatial sections, varying cosmological constant corresponds to taking different large $N$ limits because the cosmological constant depends on $N$. This is a very nontrivial task which contains $1 / N$ effect and extended AdS/CFT correspondence. Usually AdS/CFT correspondence is defined for a fixed large $N$ limit as a correspondence between low energy effective theories of coincident $N$ D branes. Thus varying $N$ means adding $\mathrm{D}$ branes to the original $N \mathrm{D}$ brane system. Also thermodynamic volume is a response from changing number of $\mathrm{D}$ branes and it can be interpreted as a chemical potential of branes in various situations. We present an explicit expression for the ABJM theory in section 3.

In addition there is another interesting interpretation of thermodynamics volume for black holes with hyperbolic spatial section, so called, hyperbolic black holes. In this case, the entropy of the black holes can be mapped to an entanglement entropy with ball shape entangling regions whose radius is given by the cosmological constant. So physical meaning of varying $N$ is changing the size of entangling region. This generalizes the thermodynamics of entanglement entropy and we introduce new quantity, 'entanglement pressure' to explain an additional term in the generalized thermodynamics. We will show details in section 3.

\section{Thermodynamics of Bulk and Boundary}

In this section we summarize and compare thermodynamics of a gravitational system and corresponding boundary theory. Let us start with a following gravity system.

$$
S=\frac{1}{16 \pi G} \int d^{D} x \sqrt{-g}\left(R+\frac{(D-1)(D-2)}{L^{2}}-\frac{1}{4} F^{M N} F_{M N}\right),
$$

where $F$ is a Maxwell field strength given by $F=d A$. This action admits an ansatz as follows:

$$
d s^{2}=-f(r) d t^{2}+r^{2} d \Omega_{\kappa}^{2}+\frac{d r^{2}}{f(r)},
$$

where the subscript $\kappa$ of the spatial section $\Omega_{\kappa}$ can be 1,0 and -1 for spherical, flat and hyperbolic spatial section, respectively. Then one can obtain the following charged black hole solution,

$$
\begin{aligned}
& f(r)=\frac{r^{2}}{L^{2}}+\kappa-\frac{16 \pi \tilde{m}}{(D-2) r^{D-3}}+\frac{G^{\frac{2(D-3)}{D-2}} \tilde{q}}{2(D-2)(D-3) r^{2(D-3)}} \\
& A=\left(16 G^{\frac{1}{D-2}} \mu-\frac{G^{\frac{D-3}{D-2}}}{(D-3) r^{D-3}} \tilde{q}\right) d t
\end{aligned}
$$

where $\tilde{m}$ and $\tilde{q}$ are mass and charge density over $\Omega_{k}$.

Now, one can define thermodynamic variables in the bulk and construct the thermodynamics of the gravitational system. First the Hawking temperature and the entropy are defined as follows:

$$
T_{H}=\frac{1}{4 \pi} e^{A\left(r_{h}\right)} f^{\prime}\left(r_{h}\right), S=\frac{1}{4 G} r_{h}^{D-2} \operatorname{Vol}_{\Omega_{\kappa}}
$$


where $r_{h}$ denotes the horizon of the black holes. The bulk pressure is obtained by contribution of the cosmological constant to the energy-momentum tensor.

$$
P_{B}=\frac{(D-1)(D-2)}{16 \pi G} .
$$

Then the Smarr relation for this system can be derived by considering $f\left(r_{h}\right)=0$ as follows:

$$
(D-3) M=(D-2) S T_{H}-2 P_{B} V_{t h}+(D-3) \mu Q,
$$

where $M$ and $Q$ are the total mass and charge for $\Omega_{k}$. Here we defined a quantity $V_{t h}$ as

$$
V_{t h}=\frac{r_{h}^{D-1}}{D-1} \operatorname{Vol}_{\Omega_{\kappa}} .
$$

Also, one can obtain the first law of thermodynamics from variation of the Smarr relation (7). It can be written in the following form:

$$
d M=T_{H} d S+V_{t h} d P_{B}+\mu d Q .
$$

From this, one can see that $V_{t h}$ is indeed playing a role of the thermodynamic volume and the mass $M$ can be identified with an enthalpy of the system.

Now, one can consider corresponding dual thermodynamics of the boundary system. Thermodynamic variables of the dual conformal field theory can be obtained by holographic renormalization [10-13]. The metric of the boundary system is easily read off from the asymptotic behavior of the geometries. It is given by

$$
d s_{b}^{2}=g_{\mu \nu}^{b} d x^{\mu} d x^{v}=-d t^{2}+L^{2} d \Omega_{\kappa}^{2} .
$$

Then the volume of the space is

$$
\mathcal{V}_{b}=L^{D-2} \operatorname{Vol}_{\Omega_{\kappa}},
$$

thus varying L correponds to varying the size of space in the dual field theory. Derivative of the onshell gravity action with respect to this boundary metric gives energy momentum tensor as follows:

$$
\left\langle T^{\mu v}\right\rangle=\epsilon u^{\mu} u^{v}+\frac{\epsilon}{D-2}\left(g_{b}^{\mu v}+u^{\mu} u^{v}\right),
$$

where $\epsilon$ and $u^{\mu}$ are energy density and a timelike normal vector of the boundary system. The energy density is $\tilde{m}$ for even $D$. Otherwise, for odd $D$, it contains Casimir contributions as follows:

$$
\epsilon=\tilde{m}+\frac{D-2}{32} L^{D-3} \kappa^{\frac{D-1}{2}}\left(\begin{array}{c}
\frac{1}{2} \\
\frac{D-1}{2}
\end{array}\right) .
$$

The Smarr relation (7) gives an expression of the euclidean on-sell action in terms of other thermodynamic variables,

$$
S^{\text {on-shell }} \frac{T_{H}}{\mathcal{V}_{b}}=\epsilon-s T_{H}-\mu \rho
$$

where $\rho$ is the energy density of the boundary system and it can be obtained by $\langle\rho\rangle=\frac{\delta S^{\text {on-shell }}}{\delta \mu}$ and $s$ is the entropy density of the boundary system. 
Also, the first law (9) can be written in terms of boundary quantities as follows:

$$
d \epsilon=T_{H} d s+\mu d \rho-\frac{1}{D-2} \frac{(\epsilon-\mu \rho)}{\mathcal{V}_{b}} d \mathcal{V}_{b}
$$

With extensive quantities, this becomes

$$
d E_{b}=T_{H} d S_{b}+\mu d Q_{b}-\left(\frac{D-3}{D-2}(\epsilon-\mu \rho)-s T_{H}\right) d \mathcal{V}_{b}
$$

where $E_{b}$ adnd $Q_{b}$ are total energy and total charge of the boundary system. Finally, we may conclude that the pressure is

$$
\mathcal{P}_{b}=\left(\frac{D-3}{D-2}(\epsilon-\mu \rho)-s T_{H}\right)
$$

and the grand potential is given by the euclidean on-shell action,

$$
\mathcal{W}=E_{b}-S T_{H}-\mu Q_{b} .
$$

In addition, there is a difference from the bulk thermodynamics. The energy of the boundary system still plays a role of internal energy rather than enthalpy. This is confirmed by the first law. Lastly we would like to point out there is an invariant quantity between bulk and boundary theories, which is pressure times volume:

$$
P_{B} V_{t h}=\mathcal{P}_{b} \mathcal{V}_{b}
$$

Therefore, the thermodynamic volum in the bulk is given by ratio of the pressures times the boundary volume. The meaning of this relation is not clear so far. However, it must be an interesting relation for the bulk-boundary duality.

\section{Physical Interpretation and Discussion}

In this final section we move on to physical interpretation of the extended thermodynamics. As we explained in section 1 . The thermodynamic volume is related to a kind of chemical potential to the number of branes for black branes and black holes. In particular, we know dual field theories for some cases exactly. $D=4$ case is for $\mathcal{N}=4$ super Yang-Mills theory and $D=3$ case can describe the ABJM theory. We consider the 4 dimensional case.

The radius of 4 dimensional AdS spacetime can be written in terms of parameters of the ABJM theory as follows:

$$
L=\frac{l_{p}}{2}\left(2^{5} \pi^{2} N k\right)^{1 / 6},
$$

where $l_{p}, N$ and $k$ are the Planck length, the rank of gauge group and the Chern-Simons level of the ABJM theory. Then the first law of the dual theory is given by

$$
d E_{b}=T_{H} d S+\mu d Q+\mu_{N} d N
$$

where $\mu_{N}$ is the chemical potential of M2 brane and it is related to thermodynamic volume. The expression is

$$
\mu_{N}=-\mathcal{P}_{b}\left(\frac{\partial \mathcal{V}_{b}}{\partial N}\right)=-P_{B} V_{t h}\left(\frac{\partial \log \mathcal{V}_{b}}{\partial N}\right)
$$


Now we discuss on the hyperbolic black hole case, i.e, black hole with hyperbolic spatial section. The relevant background is

$$
f(r)=\frac{r^{2}}{L^{2}}-1
$$

which is given by setting $\tilde{m}=\tilde{q}=0$. Then the black hole entropy corresponds to the entanglement entropy with a ball shape entangling region [14]. The radius of the entangling region is given by $L$. Thus varying $L$ is nothing but changing the size of the region for trace in entanglement entropy calculation. Thus the thermodynamic volume is related to a field theory quantity which is defined in the following generalized first law of entanglement entropy $S_{E E}$ over a region $\mathcal{A}$.

$$
\delta S_{E E}=\delta\left\langle H_{\mathcal{A}}\right\rangle+\mathcal{P}_{E E} \delta \mathcal{A}
$$

Where $H_{\mathcal{A}}$ is modular hamiltonian over $\mathcal{A}$ and $\mathcal{P}_{E E}$ is a response of entanglement entropy to varying $\mathcal{A}$.

The extended black hole thermodynamics by varying cosmological constant has many applications in the dual field theory. In this paper, we introduce several examples. More details and study would be presented in our next paper [15]. As a future direction, we may consider the thermodynamic volume in an inflation model or de Sitter space. In these cases, the thermodynamic volume is related to the slow roll parameter of inflation models and it is interesting to see if the thermodynamic volume plays a role of an effective volume describing dark matter [16] .

\section{Acknowledgement}

K.K. was supported by NRF Grant 2015R1D1A1A01058220.

\section{References}

[1] G. 't Hooft, "Dimensional reduction in quantum gravity," Salamfest 1993:0284-296 [grqc/9310026].

[2] L. Susskind, "The World as a hologram," J. Math. Phys. 36, 6377 (1995) [hep-th/9409089].

[3] J. M. Maldacena, "The Large N limit of superconformal field theories and supergravity," Int. J. Theor. Phys. 38, 1113 (1999) [Adv. Theor. Math. Phys. 2, 231 (1998)] [hep-th/9711200].

[4] E. Witten, "Anti-de Sitter space, thermal phase transition, and confinement in gauge theories," Adv. Theor. Math. Phys. 2, 505 (1998) [hep-th/9803131].

[5] D. Kastor, S. Ray and J. Traschen, "Enthalpy and the Mechanics of AdS Black Holes," Class. Quant. Grav. 26, 195011 (2009) [arXiv:0904.2765 [hep-th]].

[6] B. P. Dolan, "The cosmological constant and the black hole equation of state," Class. Quant. Grav. 28, 125020 (2011) [arXiv:1008.5023 [gr-qc]].

[7] B. P. Dolan, "Pressure and volume in the first law of black hole thermodynamics," Class. Quant. Grav. 28, 235017 (2011) [arXiv:1106.6260 [gr-qc]].

[8] B. P. Dolan, "Black holes and Boyle's law - The thermodynamics of the cosmological constant," Mod. Phys. Lett. A 30, no. 03n04, 1540002 (2015) [arXiv:1408.4023 [gr-qc]].

[9] D. Kubiznak, R. B. Mann and M. Teo, "Black hole chemistry: thermodynamics with Lambda," Class. Quant. Grav. 34, no. 6, 063001 (2017) [arXiv:1608.06147 [hep-th]].

[10] V. Balasubramanian and P. Kraus, "A Stress tensor for Anti-de Sitter gravity," Commun. Math. Phys. 208, 413 (1999) [hep-th/9902121]. 
[11] M. Henningson and K. Skenderis, “The Holographic Weyl anomaly,” JHEP 9807, 023 (1998) [hep-th/9806087].

[12] S. de Haro, S. N. Solodukhin and K. Skenderis, "Holographic reconstruction of space-time and renormalization in the AdS / CFT correspondence," Commun. Math. Phys. 217, 595 (2001) [hepth/0002230].

[13] K. Skenderis, "Lecture notes on holographic renormalization," Class. Quant. Grav. 19, 5849 (2002) [hep-th/0209067].

[14] H. Casini, M. Huerta and R. C. Myers, "Towards a derivation of holographic entanglement entropy," JHEP 1105, 036 (2011) [arXiv:1102.0440 [hep-th]].

[15] B. Ahn, K. Kim and S. Hyun, in progress.

[16] E. P. Verlinde, "Emergent Gravity and the Dark Universe," SciPost Phys. 2, no. 3, 016 (2017) [arXiv:1611.02269 [hep-th]]. 